\title{
Tumor secreted ANGPTL2 facilitates recruitment of neutrophils to the lung to promote lung pre- metastatic niche formation and targeting ANGPTL2 receptor signaling prevents metastatic disease.
}

Manish Charan

Nationwide Children's Hospital

Piyush Dravid

Nationwide Children's Hospital

Maren Cam

Nationwide Children's Hospital

Bhuvana Setty

Nationwide Children's Hospital

Ryan D Roberts

Nationwide Children's Hospital

Peter J Houghton

Greehey Children's Cancer Research Institute

Hakan Cam ( $\nabla$ hcam@cscc.edu )

Nationwide Children's Research Hospital

Research article

Keywords: Pre-metastatic niche formation, osteosarcoma, ANGPTL2, tumor microenvironment, neutrophils, ATN-161, deltaNp63, preclinical studies.

Posted Date: July 25th, 2019

DOI: https://doi.org/10.21203/rs.2.11900/v1

License: (9) This work is licensed under a Creative Commons Attribution 4.0 International License.

Read Full License 


\section{Abstract}

Abstract Background: The pre-metastatic niche (PMN) represents an abnormal microenvironment devoid of cancer cells, but favoring tumor growth. Little is known about the mechanisms that generate the PMN or their effects on host cells within metastasis-prone organs. Osteosarcoma is a malignant tumor of bone made devastating through aggressive metastatic spread primarily to the lung. Mechanisms driving this tropism for lung tissue remain elusive though likely invoke specific interactions between tumor cells and the lung microenvironment. Methods: By using spontaneous metastatic models, we investigated whether lung epithelial cells are essential for primary tumor induced neutrophil recruitment in lung and subsequently initiating PMN formation in osteosarcoma. Effects of a non-RGD-based integrin binding peptide (ATN-161) on osteosarcoma metastasis was examined in tail-vein mouse injection model. Results: Serum levels of ANGPTL2 in osteosarcoma patients are significantly higher compared to those in healthy controls. Tumor-derived ANGPTL2 stimulates lung epithelial cells, which is essential for primary tumor-induced neutrophil recruitment in lung and subsequent pre-metastatic niche formation. In addition, tumor cell-derived ANGPTL2 disrupts vascular endothelial cell-cell junctions, increases the permeability of lung capillaries, and facilitates the trans-endothelial passage of tumor cells. Furthermore, we showed that pharmaceutical inhibition of ANGPTL2 signaling by ATN-161 diminished metastatic load in lungs likely due to reduction of the lung pre-metastatic niche formation. Conclusions: Our data provide plausible evidence that activation of lung epithelial a5 $\beta 1$ receptor by ANGPTL2 induces chemokine production and neutrophil recruitment causing tumor growth-favoring lung microenvironment for incoming metastatic cancer cells. Pharmaceutical inhibition of ANGPTL2 signaling by ATN-161 diminished metastatic load suggesting possible therapeutic utility of ATN-161 in early stage osteosarcoma in children.

\section{Background}

The primary tumor selectively and actively modifies potential sites of metastasis before metastatic dissemination has even occurred $(1,2)$. Sowing the 'seeds' of metastasis requires the action of tumorsecreted factors that enable the 'soil' at distant metastatic sites to encourage the outgrowth of incoming cancer cells (3). For instance, some reports have suggested that lung epithelial cells can sense tumorderived signals that trigger pre-metastatic niche formation (reviewed in (1)). However, little is known about the regulation of lung epithelial cells by primary tumor.

Alveolar type I and type II epithelial cells serve as a biological barrier in the respiratory tract and involve in gas exchange and surface tension maintenance in the lung (4). These epithelial cells also play crucial role in the recognition of pathogen- and injury-associated signals, orchestrating innate immunity in lung to maintain pulmonary homeostasis (5). For instance, activation of various pattern-recognition receptors (PRRs) in these cells and subsequent production of cytokines and chemokines induces immune cell infiltration and inflammation (6-9). Interestingly, neutrophils, which are recruited in the metastatic niche due to inflammation, can modify their polarization state in the tumor-bearing host, switching from suppressing to promoting roles in tumor metastasis $(10,11)$. Efficient neutrophil chemoattraction and 
cytotoxicity to tumor cells is important for the control of tumor growth and metastasis (12). However, increasing evidence suggests that neutrophils can elicit a pro-metastatic inflammatory microenvironment by suppressing innate and adaptive anti-tumor immunity (13-15). For instance, neutrophils were recently shown to be the main driver of breast cancer metastasis (16) and lung cancer metastasis after UV induced inflammation and tumor secreted exosomal RNAs $(17,18)$. Together, we set out to determine the underlying mechanism how lung epithelial cells, once activated by tumor-derived factors, contribute to initiate pro-metastatic inflammatory responses and pre-metastatic niche formation following neutrophils recruitments.

ANGPTL2 protein is a secreted glycoprotein. Upon binding its ligand, the integrin alpha5beta1 (a5ß1) receptor, ANGPTL2 exerts a function on cells both through autocrine or paracrine action manner. Different roles of ANGPTL2 in physiology and pathophysiology have been described including cell motility, expression of inflammation-related genes, and MMPs (19). In contrast to induction of inflammationrelated genes by ANGPTL2, the roles of cell motility and MMPs in cancer progression are well documented (19). It is also well established that the induction of inflammation related genes result in activation of neutrophils and as described above a body of evidence suggesting that the recruitment of neutrophils promotes cancer metastasis. Collectively, by using spontaneous metastatic models, we investigated whether tumor secreted ANGPTL2 induces inflammation on lung epithelial cells by activating alpha5beta 1 (a5 $\beta 1$ ) receptor and recruitment of neutrophils resulting in pre metastatic niche formation.

\section{Methods}

\section{Patient Samples and Cell Culture}

Blood was obtained from 18 osteosarcoma patients newly diagnosed or patients with osteosarcoma currently receiving treatment at Nationwide Children's Hospital. Control blood was obtained from ten healthy patients. BD Vacutainer ${ }^{\circledR}$ EDTA blood collection tubes were used for the blood collection. Briefly, blood samples were transferred into $15 \mathrm{ml}$ conical tubes and centrifuged at $800 \mathrm{~g}$ for 7 minutes at $4 \mathrm{C}^{\circ}$. Subsequently, ANGPTL2 serum levels were analyzed by ELISA (Takara). Written informed consent for the use of blood samples was obtained from all patients and the protocol for the present study was approved by the Institutional Review Boards of the Nationwide Children's Hospital. OS17 was derived from an early passage of the OS17 patient-derived xenograft (20). OS25 and OHS cells were a gift from Oystein Fodstad's lab at the Radium Hospital in Oslo. OS17-sh $\triangle$ Np63 and OS25- $\Delta$ Np63 were described in (21). All cells were grown in RPMI supplemented with $10 \%$ FBS. K7M2 cells were obtained from ATCC (CRL-2836) and cultured as suggested. LM9 cells are a gift from Hideki Yoshikawa's lab at Osaka Medical Center for Cancer and Cardiovascular Diseases in Osaka, Japan. Murine osteosarcoma K7M2 and LM9 cells were cultured in DMEM supplemented with $10 \%$ FBS.

\section{Mice, tumor models and preclinical studies}


Conditional knockout Itga5 (integrin5a) mouse was obtained from Taconic. Sftpc-CreERT2 mice were obtained from the Jackson Laboratory. For the intratibial injections, 5x105 osteosarcoma cells were injected into the tibia of 6-8 week old mice as described in (22). After primary tumors reached around 800 $\mathrm{mm} 3$ (range from 4 to 5 weeks), primary tumor containing leg was amputated as described in (22). Eight weeks after amputation, mice were euthanized; lungs were harvested, insufflated, fixed, sectioned, and stained. Number of metastases per section was quantified. 10 mice each for the experimental and control groups were used. For the preclinical studies, 6 to 8 week old CB17-SCID mice (that retain normal NK and myeloid cell function) were inoculated via tail vein with $1 \times 106$ OS cells (day 0). Mice received ATN-161 ( $1 \mathrm{mg} / \mathrm{kg}$ I.V., 3 times/week for 6 consecutive weeks), or vehicle. For K7M2 and LM9 cells, immunocompetent BALB/c mice were used, and treatments were performed similarly as in SCID mice. Mice were monitored twice a week by measuring body weight and eBCS score as described in (23). Mice demonstrating greater than $10 \%$ body weight loss or eBCS less than 8 was euthanized by the carbon dioxide method and tissues harvested, lungs insufflated, and processed as above. Mice not demonstrating metastatic disease burden (presumably dying from other causes) were censored in the survival analysis. For the Kaplan-Meier survival analysis, 10 mice each for the experimental and control groups were used. Mice were housed in a group of up to 5 per cage in special SPF cages that included autoclaved bedding material. All mice were bred and housed at the Nationwide Children's Hospital in a specific-pathogen free facility. All animal study procedures and protocols were conducted with approval of the Nationwide Children's Hospital Institutional Animal Care and Use Committee. All sections of this report adhere to the ARRIVE Guidelines for reporting animal research (24). A completed ARRIVE guideline's checklist is included as an additional file 1.

\section{Lung Tissue Dissociation, Cell Isolation and Flow cytometry analysis}

Briefly, at the indicated time points, lung tissues were collected and cut into small pieces, and incubated in dissociation solution with $2 \mathrm{mg} / \mathrm{ml}$ collagenase type $\mathrm{l}, 2 \mathrm{mg} / \mathrm{ml}$ collagenase type IV, and $1 \mathrm{mg} / \mathrm{ml}$ DNase (Sigma). The solution was pipetted every 10 min during the incubation and suspension was dispersed through a 70-mm cell strainer. For purification of cells from lung tissues, single-cell suspensions from lungs were stained with antibodies, and T cells (CD45+ CD3+), B cells (CD45+ CD19+), macrophages (CD45+ CD11b+Gr1-), dendritic cells (CD45+ CD11c+ MHCll+), neutrophils (CD45+ CD11b+Gr1+), epithelial cells (CD45- Epcam+), and AT-II cells (CD45- SftpD+) were sorted using a flow cytometer. For flow cytometry analysis, isolated cells were incubated with anti-mouse CD16/CD32 antibody for $20 \mathrm{~min}$ at room temperature to block non-specific Fc receptor binding. Cells were then washed with PBS and stained with antibodies described above at room temperature for $20 \mathrm{~min}$. All flow cytometry analysis was performed with BD-LSR II (BD Biosciences) and data were analyzed with FlowJo software.

\section{Lentiviral production, Quantitative reverse transcription PCR (RT-qPCR), western blot and ELISA}

Specific oligonucleotides targeting ANGPTL2 (sh1: CCTGAGAGCGAGTATTATAAG, sh2:

TGGCACAACGGCAAGCAGTTC) and sh-control, (shCtr: CCTAAGGTTAAGTCGCCCTCG) were designed and 
cloned into pLKO.1 cloning vector according to the protocol recommended by Addgene (Cambridge, MA). Subsequently, lentivirus production and infections were performed as described in Addgene's pLKO.1 protocol. RNA isolation, reverse transcription and western blotting were performed similar in $(25,26)$. $\beta$ Actin antibody is from Santa Cruz and integrin-a5 antibody is from Abcam. Bv8 (Prok2), CXCL1, CXCL2, CXCL5, CXCL12 and GAPGH TaqMan ${ }^{\circledR}$ Gene Expression Assays was purchased from Applied Biosystems. ELISA kits for mouse CXCL1, CXCL2, CXCL5 and CXCL12 was purchased from R\&D Systems and assays were performed according to the manufacturer's instructions.

\section{Immunofluorescence and in vivo lung permeability assays}

HUVECs were grown to confluence on fibronectin coated chamber slides (Corning). The cells were fixed for $10 \mathrm{~min}$ in $4 \%$ paraformaldehyde and permeabilized with $0.5 \%$ Triton X-100 for 5 min. After blocking with $5 \%$ BSA, the monolayers were processed for staining with anti-beta-catenin (Santa Cruz), rhodamine phalloidin (Molecular Probes) for F-actin staining and DAPI (Vector Labs) for nuclear staining.

Fluorescence images were obtained using an Axiolmager Z1 microscopy system (Zeiss). To analyze in vivo permeability of lung blood vessels, tumor cells were infected by GFP expressing lentivirus (Cellomics Technology, Hallethorpe, MD) and inoculated into the lateral tail vein. One day post inoculation, mice were injected intravenously with rhodamine-conjugated dextran (70 kDa, Invitrogen) at $2 \mathrm{mg}$ per $20 \mathrm{~g}$ body weight. After $3 \mathrm{~h}$, mice were sacrificed; lungs were extracted and fixed by intra-tracheal injection of $5 \mathrm{~mL}$ of $4 \%$ PFA. Lungs were fixed-frozen and $10 \mu \mathrm{m}$ sections were taken to be examined by confocal microscopy for vascular leakage. Images were acquired on a Zeiss LSM 700 system. After images were obtained, a region of interest (ROI) was drawn around the GFP-labeled cells and the amount of dextran dye was quantified based on rhodamine emissions. $n=40$ cells; error bars indicate s.e.m; $p$-values calculated using the one-tailed unpaired t-test.

\section{Chromatin immunoprecipitation (ChIP) and Luciferase Assay}

ChIP assay was performed according to the manufacturer's instructions (EZ ChIP Kit; Millipore, Burlington, MA) with p63 antibody (4A4, Santa Cruz Biotechnology, Santa Cruz, CA). The $\triangle$ Np63associated chromatin was analyzed from 0S25- $\triangle \mathrm{Np} 63$ cells via quantitative real time PCR (qRT-PCR) using primer sets flanking the NF-KB binding site (Forward, ACTCAGGTGTGAAGTCACAGAG, Reverse, GCATCGGAGCTGCTCGAAGTTA). Indicated plasmids were transfected along with the corresponding luciferase reporter vector into 293T cells. The human ANGPTL2 promoter (1719bp) was cloned from human genomic DNA (BioChain institute Inc., Newark, CA) by PCR (ForwardATAGGTACCTTGCTCACGTGTCTGCGGCTG and Reverse- GATCTCGAGCCGCCAGAGGAAACTGTG) and subcloned into the pGL3 (Promega, Madison, WI) luciferase reporter vector. The ANGPTL2mutNF-KB luciferase reporter constructs were generated from the pGL3-ANGPTL2-Luc2 construct with the 
QuikChange $^{\text {TM }}$ II Site-Directed Mutagenesis Kit (Agilent, Santa Clara, CA). All luciferase assays were performed according to the manufacturer's instructions (Promega, Madison, WI).

\section{Statistical analysis}

Data was graphed and analyzed using Graphpad Prism 7 (GraphPad Software). Error bars represent mean \pm SD from triplicate measurements from one experiment. One representative experiment is showing. Differences between two groups were analyzed by unpaired Student's t-test. The numbers of lung section with metastatic nodules were compared either with an ordinary Two-way Anova analysis, or the Mann-Whitney $U$ test as described in figure legends. Survival curves were plotted using the KaplanMeier method, and log-rank tests were used to compare curves between groups.

\section{Results}

High level of ANGPTL2 secretion plays a critical role in osteosarcoma metastasis.

Given the known actions of ANGPTL2 and the strong propensity of osteosarcoma for metastasis to lung, we asked whether ANGPTL2 might involve in osteosarcoma metastasis. Interestingly, we found that ANGPTL2 levels were strikingly elevated in serum from osteosarcoma patients compared to healthy controls (Figure 1A). We suspected that high level ANGPTL2 secretion might endow these childhood tumors with greater metastatic potential. We first asked whether high expression of ANGPTL2 in osteosarcoma cell lines correlated with metastatic nodule formation in vivo. We found that the OS17 cell line, which secretes high levels of ANGPTL2, formed considerably more lung metastases than OS 25 cells, which secrete lower ANGPTL2 (Supplementary Fig. 1A-B). To test the role of ANGPTL2 in metastasis development, we knocked down ANGPTL2 gene expression in highly metastatic mouse (LM9, K7M2) and human (OS17) osteosarcoma cell lines and verified knockdown efficiency by ELISA (Figure 1B). We then implanted these osteosarcoma cells (with or without ANGPTL2 knockdown) into the tibia of syngeneic (LM9, K7M2) or SCID mice to generate orthotopic tumors and determined serum levels of ANGPTL2 after 2 weeks. Similar to our observations in patients, serum from mice injected with non-manipulated tumor cells (control shRNA) showed high levels of ANGPTL2 (Figure 1C). However, serum ANGPTL2 levels were dramatically lower in mice bearing ANGPTL2-suppressed tumor cells. In a separate experiment, the same cell lines (with or without ANGPTL2 knockdown) were inoculated into the tibia, let to grow to a predetermined size, and then removed by limb amputations. Eight weeks later lung metastases were evaluated (used animal models are described in Supplementary Fig. 2). As shown in Figure 1D, downregulation of ANGPTL2 expression significantly reduced lung metastasis compared to control cells, confirming a functional role for ANGPTL2 in the development of spontaneous lung metastasis. In contrast, primary tumor growth rates for LM9, K7M2 and OS17 primary tumors were unaffected by downregulation of ANGPTL2 (Supplementary Fig. 1C).

\section{ANGPL2 receptor integrin a5 $\beta 1$ required for the pre-metastatic niche formation.}


Next, to obtain an overall view of ANGPTL2's receptor integrin a5 $\beta 1$ in the metastatic process, we crossed Itga5 (integrin5a) conditional knockout mice (Taconic) with Sftpc-CreERT2 (Jackson Laboratory) to induce time- and tissue-specific knockout of integrin a5 gene in Type II alveolar cells (herein, Itga5-floxed, after tamoxifen administration). Of note, previous research suggested that alveolar type II cells possess the ability of initiating lung tumor development (27). Subsequently, we isolated the alveolar type II (AT-II) cells from Itga5-floxed mice and the integrin a5 gene knockout was verified by western blotting (Figure $2 A)$. To assess the role of ANGPTL2 receptor integrin a5 $\beta 1$ in the pre-metastatic niche formation, Itga5floxed mice were inoculated with LM9 or K7M2 osteosarcoma cells into tibia. After these tumors reached a pre-determined size, these limbs were amputated and then observed for signs of lung metastasis. As shown in Figure 2B, we found that Itga5-floxed mice showed significant reduction in lung metastasis compared with integrin a5wild-type (WT) littermates. Furthermore, Itga5-floxed mice survived much longer than WT littermates after tumor removal (Figure 2C-D). However, no difference was observed in early primary tumor growth in these mouse models (Figure 2E-F). Taken together, these observations indicate that deletion of integrin a5 $\beta 1$ in the alveolar type II (AT-II) cells impair osteosarcoma lung colonization, but not the growth of primary tumors in the bone.

\section{Deletion of integrin a5 31 receptor in AT-II cells attenuates accumulation of neutrophils to the lung}

We next examined the downstream signaling pathways that support or initiate pre-metastatic niche following integrin a5 $\beta 1$ receptor activation by ANGPTL2 in AT-Il cells. We therefore evaluated whether the activation of integrin a5 $\beta 1$ by ANGPTL2 impacted neutrophil recruitment to the lung under physiological conditions. Accordingly, we examined the cellular composition of the lung in Itga5-floxed and WT littermates at days 1, 7 and 14 after intratibial inoculation with LM9 and K7M2 cells as described in materials and methods. As shown in Figure 3A-B, CD11b+ myeloid cells were remarkably expanded in the pre-metastatic lung, among which CD45+ CD11b+Gr1+ neutrophils were the most enriched in WT mice compared to macrophages and dendritic cells (Supplementary Fig 4A-D). Both the percentage and the number of neutrophils in pre-metastatic lung were significantly reduced in Itga5-floxed mice compared with WT littermates (Figures 3A-B). Our data indicate that neutrophils are evident in pre-metastatic niche formation. Of note, there was still an increase of neutrophils in the Itga5-floxed mice upon tumor inoculation (Figures 3A-B), suggesting that other pathways also play minor roles in the recruitment of neutrophils to the premetastatic niche. Moreover, we evaluated BV8 expression, a chemokine that is highly expressed in pro-metastatic neutrophils, and described as one of the major components in the metastatic niche (28). As shown in Supplementary Figure 4E, much higher expression of Bv8 in neutrophils than in macrophages or dendritic cells was detected in tumor-bearing mice, further supporting our finding that neutrophils are the most prominent in pre-metastatic niche formation.

\section{Activation of Integrin a5 $\beta 1$ receptor by ANGPTL2 induces chemokine production and neutrophil recruitment}

Next, to confirm whether ANGPTL2 activates integrin a5 $\beta 1$ receptor leading to neutrophils recruitments, we injected LM9-shCtr, LM9-shANGPTL2, K7M2-shCtr and K7M2-shANGPTL2 cells into tibia of WT mice. 
As shown in Figure 3C, the absolute number of neutrophils in the lung were significantly lower in ANGPTL2 depleted tumor cells verifying essential role of tumor secreted ANGPTL2 in neutrophils recruitments. To dissect the underlying mechanisms of impaired neutrophil recruitment in tumor-bearing Itga5-floxed mice further, we first analyzed the levels of some the important chemokines (CXCL1, CXCL2, CXCL5, and CXCL12), which are known to be required for neutrophil chemotaxis. In a separate experiment, the serum and bronchoalveolar lavage fluid (BALF) were isolated one week after tibia injections and analyzed with ELISA. As shown in Figure 3D, the secretion of crucial chemokines in the serum was significantly reduced in tumor-bearing Itga5-floxed animals. Moreover, we found that integrin a5 $\beta 1$ deficiency in alveolar type II reduces cytokine expression (Figure 3E). Taken together, our results suggest that the activation of lung epithelial a $5 \beta 1$ receptor by ANGPTL2 induces chemokine production and neutrophil recruitment causing tumor growth-favoring lung microenvironment for incoming metastatic cancer cells. In a final experiment, we sought to demonstrate that activated neutrophils were necessary for the pre-metastatic niche formation and establishment of metastasis in osteosarcoma. Neutrophils were depleted by i.p. injection of anti-Ly6G $(0.1 \mathrm{mg})$ monoclonal antibody (Clone 1A8), starting 3 days prior to inoculating and ongoing twice a week for 10 weeks. IgG2a (Clone 2A3) was used as an isotype matched control treatment. LM9 and K7M2 osteosarcoma cells were injected into the tibia of WT mice, 3 days after the initial anti-Ly $6 G$ antibody dose ( $n=40,10$ mice for each group). The leg with primary tumor was amputated at 4-5 weeks after tumor cell inoculation, and animals were euthanized after four weeks. The numbers of lung section with metastatic nodules were compared between groups. As shown in Figure 3F, depletion of neutrophils in tumor bearing animals had significant effects on lung metastasis. Together, these results confirm further that neutrophils are critical for pre-metastatic niche formation in these osteosarcoma models. Tumor-secreted ANGPTL2 promotes osteosarcoma extravasation.

After a successful establishment of pre metastatic niche formation, circulating cancer cells need to disrupt lung capillary endothelial junctions to selectively seed lung metastasis, an event described as extravasation. Previous studies showed that the induction of angiopoietin like-4 (ANGPTL4), a member of angiopoietins, increased extravasation of breast tumor cells in the lung. Consequently, we investigated whether ANGPTL2 affects endothelial cell layers in a manner that would facilitate the passage of tumor cells across endothelia. Human vascular endothelial cells (HUVEC) were allowed to grow to form tight monolayers on tissue culture dishes. At this point, the monolayers were exposed to media containing human recombinant ANGPTL2, control media (Figure 4A), or media conditioned by control OS17 osteosarcoma cells or by ANGPTL2-depleted OS17 cells (Figure 4B). In each case ANGPTL2 caused an acute disruption of endothelial cell-cell junctions. Staining with antibodies against the adherens junction component $\beta$-catenin, or staining of the actin cytoskeleton with phalloidin, revealed that the monolayer integrity was dramatically perturbed by ANGPTL2 (Figures 4A and B). We also evaluated the ability of ATN-161 (a selective small peptide antagonist of integrin a5 $\beta 1$ ) to rescue the disruption of endothelial junctions. As shown in Figure 4C, HUVEC monolayers that were exposed to media conditioned by OS17 cells showed significant disruption of endothelial junctions. However, administration of ATN-161 in the conditioned media clearly rescued the disruption of endothelial junctions (Figure 4C) suggesting that 
targeting ANGPTL2 receptor by ATN-161 might prevent emergence of pulmonary metastasis and prolong survival in osteosarcoma models as explored below. To demonstrate vessel permeability in vivo, GFPlabeled OS17 cells either expressing shRNA targeting ANGPTL2 or scramble (control) were inoculated into SCID mice. One day post inoculation, the same animals were injected with rhodamine-conjugated dextran. Subsequently, the lungs were removed and investigated for retained rhodamine by using fluorescent microscopy. As shown in Figure 4D, regardless of ANGPTL2 knockdown, all injected OS17 cells lodged in to the lungs (green fluorescent). However, only OS17, overexpressing ANGPTL2, significantly induced vessel permeability, which was evident by rhodamine signal (red fluorescent, Figure 4D). Together, around 5 -fold increase in surrounding rhodamine signal were determined by quantitative analysis of the fluorescent area (Figure 4E). Lastly, to test the effect of ANGPTL2 on cell migration across an endothelial layer, endothelial monolayers were set on transwell tissue culture inserts. OS17 cells overexpressing ANGPTL2 passed twice as efficiently through these layers into the lower chamber of the transwell compared to OS17-shANGPTL2 cells (Figure 4F). In summary, our results revealed that ANGPTL2 disrupts the integrity of vascular endothelial cell layers both in vitro and in the lungs, facilitating the passage of osteosarcoma cancer cells.

\section{$\Delta$ Np63 drives ANGPTL2 expression through interacting NF-KB binding site.}

We next examined the underlying molecular mechanism of high levels of ANGPTL2 secretion in osteosarcoma. We and others demonstrated that osteosarcoma xenografts, cell lines and patient samples express high-levels of $\Delta \mathrm{Np} 63(25,29,30)$. To interrogate the role of $\Delta \mathrm{Np} 63$ in ANGPTL2 regulation in osteosarcoma, we first utilized our OS17-sh $\triangle$ Np63 and OS25- $\triangle$ Np63 cell lines expressing high or low basal levels of this gene (21). As shown in Figures 5A-B, both mRNA and secretion level of ANGPTL2 in osteosarcoma cells depend on $\triangle N$ p63 expression. Subsequently, we wondered whether $\triangle N$ n63 expressing primary tumors in the bone milieu would indeed secrete detectable ANGPTL2 levels in an in vivo orthotopic mouse model. Therefore, luciferase-expressing osteosarcoma cells were injected into the tibia of SCID mice (Figure 5C-D) and serum samples were isolated from the blood of tumor bearing animals after 4 weeks. As shown in Fig. 5E, we found that ANGPTL2 levels in serum is significantly correlated with $\triangle \mathrm{Np} 63$ expression in tibial tumors. Furthermore, to validate our findings, we examined whether $\triangle N p 63$ can activate the ANGPTL2 promoter in vitro. To this end, we cloned the human ANGPTL2 promoter (1716bp) into a luciferase reporter plasmid. As shown in Fig. 5F, $\triangle N p 63$ enhances the ANGPTL2 promoter activity in a dose-dependent manner. The ANGPTL2 promoter has been studied, and binding several transcription factors confirmed (31). While $\triangle N p 63$ lacks a transcriptional activation domain and is therefore transcriptionally incompetent, it still harbors a DNA binding domain. It is conceivable that $\triangle N p 63$ recruits another transcription factor to the binding site in the ANGPTL2 promoter, thereby inducing its expression. For example, previous study demonstrated that $\triangle N p 63$, RelA, and cRel members of the NF-KB family could interact together to affect the transcription of NF-KB/Rel target genes (32). To test whether $\triangle N p 63$ similarly binds ANGPTL2 promoter region, which contains known NF-kB/Rel regulatory elements, we first conducted ChIP assay with primer pairs covering NF-KB binding site on ANGPTL2 promoter. As shown in Figure $5 \mathrm{H}$, binding of $\triangle$ Np63 to NF-KB site on ANGPTL2 promoter suggests direct regulation of ANGPTL2 by $\triangle N$ p63. To support this finding further, we co-transfected 
$\triangle$ Np63 with ANGPTL2 reporter construct either containing wild type or mutated NF-KB-binding site. As shown in Figure $5 \mathrm{G}, \triangle \mathrm{Np} 63$ was able to increase the transactivation of the wild type ANGPTL2 promoter, however only a minimal increase in transactivation of the NF-KB mutated promoter was observed. Together, these results demonstrated that $\triangle N p 63$ drives ANGPTL2 expression via NF-KB binding site in osteosarcoma.

\section{Targeting ANGPTL2 receptor signaling prevents the formation of lung metastasis in preclinical studies.}

We lastly wondered whether pharmacologic intervention of ANGPTL2 receptor (a5 $\beta 1$ ) has an effect on metastatic burden in an in vivo metastasis mouse model. We utilized the non-RGD-based integrin binding peptide (ATN-161), which has advanced to clinical trials (clinicaltrials.gov). ATN-161 is well tolerated, and has been investigated in a phase 1 trials alone or in combination with chemotherapy in adult patients with solid tumors (33). As shown in Figure 6A-C, animals treated with ATN-161 developed significantly less numbers of metastatic foci compared to the vehicle treated animals. Importantly, our survival studies demonstrated that targeting ANGPTL2 receptor by ATN-161 prolonged overall survival in osteosarcoma models Figure 6D, thereby laying the groundwork for future clinical trials in children affected with this devastating disease.

\section{Discussion}

ANGPTL2 is a mediator of chronic inflammation and inflammatory carcinogenesis. Here, we report that ANGPTL2 secretion is elevated in osteosarcoma patients when compared to healthy donors. The biological and clinical significance of ANGPTL2 remains unknown in human cancer. Nevertheless, recent studies have shown ANGPTL2 as a potential serum biomarker for colorectal (34), gastric (35) and esophageal cancer (36). While steady progress has been made in the identification of genetic alterations in osteosarcoma, no individual molecular marker has thus far been demonstrated to have a better prognostic significance in the treatment of osteosarcomas than the current clinical markers (37). Therefore, novel biomarkers are needed to stratify patients and to enhance existing techniques to interrogate treatment response, for outcome to improve. Future studies will reveal whether ANGPTL2 in serum acts as a biomarker for osteosarcoma progression.

The pre-metastatic niche represents an abnormal microenvironment that while devoid of cancer cells favors tumor growth. Little is known about the mechanisms involved and how these impact host cells in pre-metastatic organs. Recognition and sensing of primary tumor-derived signals to initiate subsequent immune responses in distinct organs is a critical, but poorly defined process in pre-metastatic niche formation. Here, we investigated whether lung epithelial cells activated by tumor secreted ANGPTL2 might be essential for primary tumor-induced neutrophil recruitment in lung and subsequently initiating pre-metastatic niche formation in osteosarcoma. Our study strongly suggests that tumor secreted ANGPTL2 leads to neutrophil recruitment to the lung, and is necessary for the lung colony development in spontaneous models of osteosarcoma pulmonary metastasis. It is also likely that bone marrow-derived cells are mobilized by primary tumor-derived soluble factors to the distinct organ, leading to the formation 
of a pre-metastatic niche in preparation for the settlement and further colonization of the metastatic cells. In addition to the contribution of tumor-secreted factors to pre-metastatic niche formation, there are other tumor-independent pathological and physiological processes need to be considered, such as the effects of surgery, infection and ageing, which alters the local milieu and help to create a microenvironment that is sufficiently receptive to colonization by circulating tumor cells $(38,39)$. Together, our results should be interpreted with caution.

Lung vascular endothelial junctions act as a barrier that restricts the passage of cells. Further its role in pre metastatic nice formation, we also identified that ANGPTL2 antagonizes vascular endothelial tight junctions and adherens junctions, and disrupts the integrity of capillary walls when secreted from osteosarcoma cells. These results strongly suggest that ANGPTL2 acts as an enhancer of osteosarcoma cell extravasation by transiently suppressing the integrity of capillaries and fit with the angiopoietin and angiopoietin-like factors in vascular remodeling (40-43). We further determined that $\Delta \mathrm{Np} 63$, an oncogenic isoform of p63, drives ANGPTL2 gene expression and its subsequent secretion from the osteosarcoma cells. We identified that NF-KB binding elements on ANGPTL2 promoter is crucial for $\triangle$ Np63-mediated transcriptional activities. Furthermore, we explored the therapeutic effects of ANGPTL2 receptor inhibition by integrin binding peptide (ATN-161) in preclinical studies. We revealed that ATN-161 treatment significantly inhibited lung metastasis and prolonged survival suggesting possible therapeutic use of ATN-161 in early stage osteosarcoma in children.

\section{Conclusions}

The mechanisms underlying the contributions of the primary tumor to pre-metastatic niche formation (PMN) are not fully understood. In this study, we investigated by using spontaneous metastatic models whether lung epithelial cells might be essential for primary tumor induced neutrophil recruitment in lung and subsequently initiating PMN formation in osteosarcoma. We demonstrated that ANGPTL2 played a vital role in the progression of osteosarcoma metastasis by inducing pre-metastatic lung formation and extravasation. Furthermore, our preclinical data suggested that ANGPTL2 signaling might be a potential target for the treatment of osteosarcoma metastasis. Together, results generated from our studies will have an impact not only in osteosarcoma, but other tumors in which ANGPTL2 signaling is known to be deregulated.

\section{Abbreviations}

PMN, pre-metastatic niche, ANGPTL2, angiopoietin like 2, a5 31 , integrin alpha5beta 1 , ATN-161, a nonRGD-based integrin binding peptide antagonist of integrin alpha5beta1, AT-II, alveolar type II cells, $\Delta N p 63$, an oncogenic isoform of TP63, NF-KB, nuclear factor kappa-light-chain-enhancer of activated B cells.

\section{Declarations}

\section{Ethics approval and consent to participate}


All samples were obtained with patients' informed consent. The informed consent obtained by written. This study was approved by the institutional review board/ethics committee of the Nationwide Children's Research Hospital.

\section{Consent for publication}

Not applicable.

\section{Availability of data and material}

Plasmids and generated cell lines during the current study are available from the corresponding author on request.

\section{Competing interests}

The authors declare no potential conflicts of interest

\section{Funding}

This work was supported by grants from Hyundai Hope On Wheels from Hyundai, P01CA165995 and K08CA201638 (R.D. Roberts) from the National Cancer Institute, CancerFree KIDS and by start-up funds from the Nationwide Children's Hospital. The funding bodies had no influence on the design of the study and collection, analysis, and interpretation of data and in writing the manuscript.

\section{Authors' Contributions}

Manish C., Maren C., and HC designed the study and wrote the manuscript. Manish C., Maren C. and PD performed and analyzed the in vitro and in vivo experiments. BS managed IRB studies. RDR, PJH and HC critically reviewed the manuscript. All authors read and approved the final manuscript.

\section{Acknowledgements}

We would like to thank Nationwide Children's Hospital's Morphology Core for the preparation of lung tissue slides and Flow Cytometry Core for the assisting with the flow cytometry analysis.

\section{References}

1. Peinado, H., Zhang, H., Matei, I. R., Costa-Silva, B., Hoshino, A., Rodrigues, G., Psaila, B., Kaplan, R. N., Bromberg, J. F., Kang, Y., Bissell, M. J., Cox, T. R., Giaccia, A. J., Erler, J. T., Hiratsuka, S., Ghajar, C. M., and Lyden, D. (2017) Pre-metastatic niches: organ-specific homes for metastases. Nat Rev Cancer $17,302-317$

2. Kaplan, R. N., Riba, R. D., Zacharoulis, S., Bramley, A. H., Vincent, L., Costa, C., MacDonald, D. D., Jin, D. K., Shido, K., Kerns, S. A., Zhu, Z., Hicklin, D., Wu, Y., Port, J. L., Altorki, N., Port, E. R., 
Ruggero, D., Shmelkov, S. V., Jensen, K. K., Rafii, S., and Lyden, D. (2005) VEGFR1-positive haematopoietic bone marrow progenitors initiate the pre-metastatic niche. Nature 438, 820-827

3. Sceneay, J., Smyth, M. J., and Moller, A. (2013) The pre-metastatic niche: finding common ground. Cancer Metastasis Rev 32, 449-464

4. Wagner, W. R., and Griffith, B. P. (2010) Reconstructing the lung. Science 329, 520-522

5. Whitsett, J. A., and Alenghat, T. (2015) Respiratory epithelial cells orchestrate pulmonary innate immunity. Nat Immunol 16, 27-35

6. Hartl, D., Latzin, P., Hordijk, P., Marcos, V., Rudolph, C., Woischnik, M., Krauss-Etschmann, S., Koller, B., Reinhardt, D., Roscher, A. A., Roos, D., and Griese, M. (2007) Cleavage of CXCR1 on neutrophils disables bacterial killing in cystic fibrosis lung disease. Nat Med 13, 1423-1430

7. Juncadella, I. J., Kadl, A., Sharma, A. K., Shim, Y. M., Hochreiter-Hufford, A., Borish, L., and Ravichandran, K. S. (2013) Apoptotic cell clearance by bronchial epithelial cells critically influences airway inflammation. Nature 493, 547-551

8. Brody, J. S., and Steiling, K. (2011) Interaction of cigarette exposure and airway epithelial cell gene expression. Annu Rev Physiol 73, 437-456

9. Takahashi, H., Ogata, H., Nishigaki, R., Broide, D. H., and Karin, M. (2010) Tobacco smoke promotes lung tumorigenesis by triggering IKKbeta- and JNK1-dependent inflammation. Cancer Cell 17, 89-97

10. Fridlender, Z. G., Sun, J., Kim, S., Kapoor, V., Cheng, G., Ling, L., Worthen, G. S., and Albelda, S. M. (2009) Polarization of tumor-associated neutrophil phenotype by TGF-beta: "N1" versus "N2" TAN. Cancer Cell 16, 183-194

11. Liu, Y., and Cao, X. (2016) Immunosuppressive cells in tumor immune escape and metastasis. J Mol Med (Berl) 94, 509-522

12. Granot, Z., Henke, E., Comen, E. A., King, T. A., Norton, L., and Benezra, R. (2011) Tumor entrained neutrophils inhibit seeding in the premetastatic lung. Cancer Cell 20,300-314

13. Coffelt, S. B., Kersten, K., Doornebal, C. W., Weiden, J., Vrijland, K., Hau, C. S., Verstegen, N. J. M., Ciampricotti, M., Hawinkels, L., Jonkers, J., and de Visser, K. E. (2015) IL-17-producing gammadelta T cells and neutrophils conspire to promote breast cancer metastasis. Nature 522, 345-348

14. Cools-Lartigue, J., Spicer, J., McDonald, B., Gowing, S., Chow, S., Giannias, B., Bourdeau, F., Kubes, P., and Ferri, L. (2013) Neutrophil extracellular traps sequester circulating tumor cells and promote metastasis. J Clin Invest

15. Wu, C. F., Andzinski, L., Kasnitz, N., Kroger, A., Klawonn, F., Lienenklaus, S., Weiss, S., and Jablonska, J. (2015) The lack of type I interferon induces neutrophil-mediated pre-metastatic niche formation in the mouse lung. Int $\mathrm{J}$ Cancer 137, 837-847 
16. Wculek, S. K., and Malanchi, I. (2015) Neutrophils support lung colonization of metastasisinitiating breast cancer cells. Nature 528, 413-417

17. Bald, T., Quast, T., Landsberg, J., Rogava, M., Glodde, N., Lopez-Ramos, D., Kohlmeyer, J., Riesenberg, S., van den Boorn-Konijnenberg, D., Homig-Holzel, C., Reuten, R., Schadow, B., Weighardt, H., Wenzel, D., Helfrich, I., Schadendorf, D., Bloch, W., Bianchi, M. E., Lugassy, C., Barnhill, R. L., Koch, M., Fleischmann, B. K., Forster, I., Kastenmuller, W., Kolanus, W., Holzel, M., Gaffal, E., and Tuting, T. (2014) Ultraviolet-radiation-induced inflammation promotes angiotropism and metastasis in melanoma. Nature 507, 109-113

18. Liu, Y., Gu, Y., Han, Y., Zhang, Q., Jiang, Z., Zhang, X., Huang, B., Xu, X., Zheng, J., and Cao, X. (2016) Tumor Exosomal RNAs Promote Lung Pre-metastatic Niche Formation by Activating Alveolar Epithelial TLR3 to Recruit Neutrophils. Cancer Cell 30, 243-256

19. Kadomatsu, T., Endo, M., Miyata, K., and Oike, Y. (2014) Diverse roles of ANGPTL2 in physiology and pathophysiology. Trends Endocrinol Metab 25, 245-254

20. Houghton, P. J., Morton, C. L., Tucker, C., Payne, D., Favours, E., Cole, C., Gorlick, R., Kolb, E. A., Zhang, W., Lock, R., Carol, H., Tajbakhsh, M., Reynolds, C. P., Maris, J. M., Courtright, J., Keir, S. T., Friedman, H. S., Stopford, C., Zeidner, J., Wu, J., Liu, T., Billups, C. A., Khan, J., Ansher, S., Zhang, J., and Smith, M. A. (2007) The pediatric preclinical testing program: description of models and early testing results. Pediatr Blood Cancer 49, 928-940

21. Gross, A. C., Cam, H., Phelps, D. A., Saraf, A. J., Bid, H. K., Cam, M., London, C. A., Winget, S. A., Arnold, M. A., Brandolini, L., Mo, X., Hinckley, J. M., Houghton, P. J., and Roberts, R. D. (2018) IL-6 and CXCL8 mediate osteosarcoma-lung interactions critical to metastasis. JCI Insight 3

22. Chaffee, B. K., and Allen, M. J. (2013) A clinically relevant mouse model of canine osteosarcoma with spontaneous metastasis. In Vivo 27, 599-603

23. Khanna, C., Prehn, J., Yeung, C., Caylor, J., Tsokos, M., and Helman, L. (2000) An orthotopic model of murine osteosarcoma with clonally related variants differing in pulmonary metastatic potential. Clin Exp Metastasis 18, 261-271

24. Kilkenny, C., Browne, W. J., Cuthill, I. C., Emerson, M., and Altman, D. G. (2010) Improving bioscience research reporting: the ARRIVE guidelines for reporting animal research. PLoS Biol 8 , e1000412

25. Bid, H. K., Roberts, R. D., Cam, M., Audino, A., Kurmasheva, R. T., Lin, J., Houghton, P. J., and Cam, H. (2014) DeltaNp63 promotes pediatric neuroblastoma and osteosarcoma by regulating tumor angiogenesis. Cancer Res 74, 320-329

26. Cam, M., Bid, H. K., Xiao, L., Zambetti, G. P., Houghton, P. J., and Cam, H. (2014) p53/TAp63 and AKT regulate mammalian target of rapamycin complex 1 (mTORC1) signaling through two independent parallel pathways in the presence of DNA damage. J Biol Chem 289, 4083-4094

27. Lin, C., Song, H., Huang, C., Yao, E., Gacayan, R., Xu, S. M., and Chuang, P. T. (2012) Alveolar type II cells possess the capability of initiating lung tumor development. PLoS One 7, e53817 
28. Kowanetz, M., Wu, X., Lee, J., Tan, M., Hagenbeek, T., Qu, X., Yu, L., Ross, J., Korsisaari, N., Cao, T., Bou-Reslan, H., Kallop, D., Weimer, R., Ludlam, M. J., Kaminker, J. S., Modrusan, Z., van Bruggen, N., Peale, F. V., Carano, R., Meng, Y. G., and Ferrara, N. (2010) Granulocyte-colony stimulating factor promotes lung metastasis through mobilization of Ly6G+Ly6C+ granulocytes. Proc Natl Acad Sci U S A 107, 21248-21255

29. Cam, M., Gardner, H. L., Roberts, R. D., Fenger, J. M., Guttridge, D. C., London, C. A., and Cam, H. (2016) DeltaNp63 mediates cellular survival and metastasis in canine osteosarcoma. Oncotarget 7, 48533-48546

30. Ram Kumar, R. M., Betz, M. M., Robl, B., Born, W., and Fuchs, B. (2014) DeltaNp63alpha enhances the oncogenic phenotype of osteosarcoma cells by inducing the expression of GLI2. BMC Cancer 14, 559

31. Thorin-Trescases, N., and Thorin, E. (2014) Angiopoietin-like-2: a multifaceted protein with physiological and pathophysiological properties. Expert Rev Mol Med 16, e17

32. Yang, X., Lu, H., Yan, B., Romano, R. A., Bian, Y., Friedman, J., Duggal, P., Allen, C., Chuang, R., Ehsanian, R., Si, H., Sinha, S., Van Waes, C., and Chen, Z. (2011) DeltaNp63 versatilely regulates a Broad NF-kappaB gene program and promotes squamous epithelial proliferation, migration, and inflammation. Cancer Res 71, 3688-3700

33. Cianfrocca, M. E., Kimmel, K. A., Gallo, J., Cardoso, T., Brown, M. M., Hudes, G., Lewis, N., Weiner, L., Lam, G. N., Brown, S. C., Shaw, D. E., Mazar, A. P., and Cohen, R. B. (2006) Phase 1 trial of the antiangiogenic peptide ATN-161 (Ac-PHSCN-NH(2)), a beta integrin antagonist, in patients with solid tumours. Br J Cancer 94, 1621-1626

34. Yoshinaga, T., Shigemitsu, T., Nishimata, H., Kitazono, M., Hori, E., Tomiyoshi, A., Takei, T., and Yoshida, M. (2015) Angiopoietin-like protein 2 as a potential biomarker for colorectal cancer. Mol Clin Oncol 3, 1080-1084

35. Yoshinaga, T., Shigemitsu, T., Nishimata, H., Takei, T., and Yoshida, M. (2015) Angiopoietin-like protein 2 is a potential biomarker for gastric cancer. Mol Med Rep 11, 2653-2658

36. Ide, S., Toiyama, Y., Shimura, T., Kawamura, M., Yasuda, H., Saigusa, S., Ohi, M., Tanaka, K., Mohri, Y., and Kusunoki, M. (2015) Angiopoietin-Like Protein 2 Acts as a Novel Biomarker for Diagnosis and Prognosis in Patients with Esophageal Cancer. Annals of surgical oncology 22, 2585-2592

37. Kong, C., and Hansen, M. F. (2009) Biomarkers in Osteosarcoma. Expert Opin Med Diagn 3, 1323

38. Shibue, T., and Weinberg, R. A. (2011) Metastatic colonization: settlement, adaptation and propagation of tumor cells in a foreign tissue environment. Semin Cancer Biol 21, 99-106

39. Weilbaecher, K. N., Guise, T. A., and McCauley, L. K. (2011) Cancer to bone: a fatal attraction. Nat Rev Cancer 11, 411-425

40. Camenisch, G., Pisabarro, M. T., Sherman, D., Kowalski, J., Nagel, M., Hass, P., Xie, M. H., Gurney, A., Bodary, S., Liang, X. H., Clark, K., Beresini, M., Ferrara, N., and Gerber, H. P. (2002) ANGPTL3 
stimulates endothelial cell adhesion and migration via integrin alpha vbeta 3 and induces blood vessel formation in vivo. J Biol Chem 277, 17281-17290

41. Gale, N. W., Thurston, G., Hackett, S. F., Renard, R., Wang, Q., McClain, J., Martin, C., Witte, C., Witte, M. H., Jackson, D., Suri, C., Campochiaro, P. A., Wiegand, S. J., and Yancopoulos, G. D. (2002) Angiopoietin-2 is required for postnatal angiogenesis and lymphatic patterning, and only the latter role is rescued by Angiopoietin-1. Dev Cell 3, 411-423

42. Parikh, S. M., Mammoto, T., Schultz, A., Yuan, H. T., Christiani, D., Karumanchi, S. A., and Sukhatme, V. P. (2006) Excess circulating angiopoietin-2 may contribute to pulmonary vascular leak in sepsis in humans. PLoS Med 3, e46

43. Padua, D., Zhang, X. H., Wang, Q., Nadal, C., Gerald, W. L., Gomis, R. R., and Massague, J. (2008) TGFbeta primes breast tumors for lung metastasis seeding through angiopoietin-like 4. Cell 133, 66-77

\section{Figures}

\section{Figure 1}

A

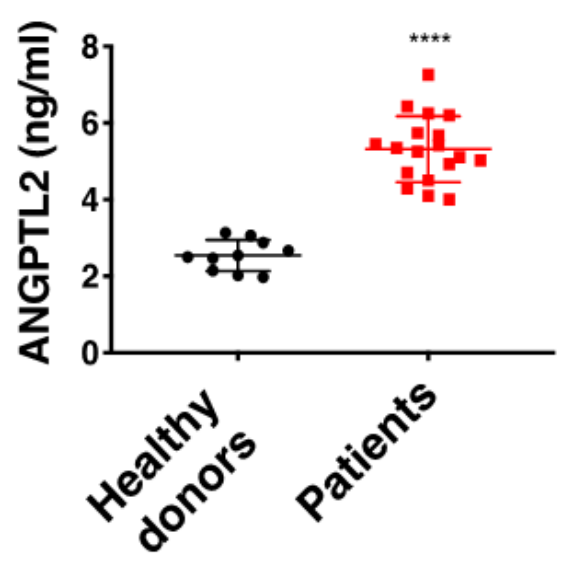

C

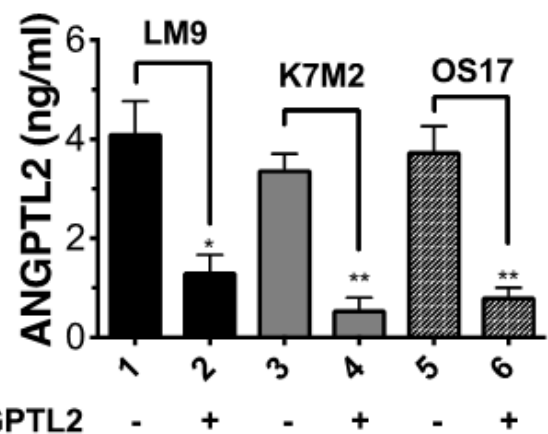

shANGPTL2
B

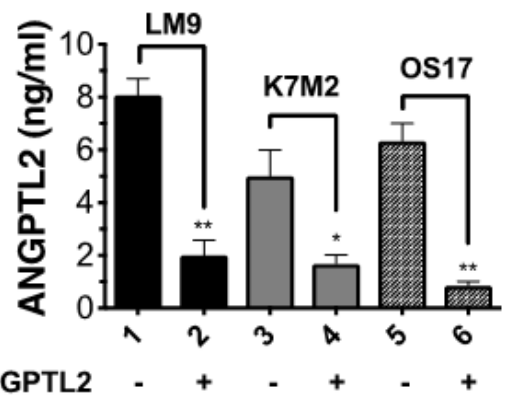

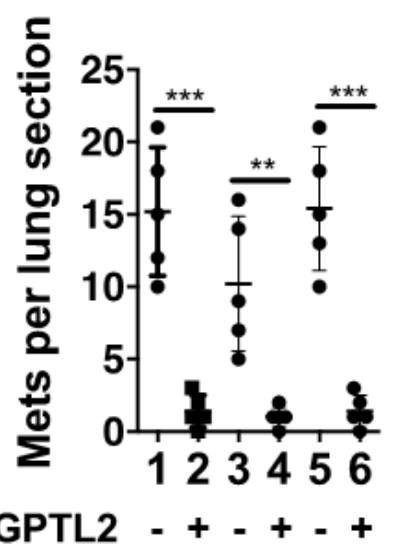


Figure 1

ANGPTL2 plays a crucial role in osteosarcoma metastasis. A. Osteosarcoma patients have high levels of serum ANGPTL2. Serum samples from osteosarcoma patients were obtained at varying stages of osteosarcoma therapy and ANGPTL2 levels were compared to healthy donors. Statistical analyses were performed using GraphPad Prism software. Results are expressed as mean $\pm S D$. ${ }^{\star} \star \star \star P<0.0001$ versus healthy donors using unpaired Student's t-tests with Welch's correction. B. ANGPTL2 knockdown efficiency in osteosarcoma cells was tested by ELISA as described in Materials and Methods. C. ANGPTL2 levels in serum from tumor-bearing animals ( \pm ANGPTL2 knockdown) were determined after 14-day tumor growth using an ANGPTL2 ELISA kit. D. ANGPTL2 knockdown reduces metastatic lung colonization in the spontaneous metastatic mouse model as described in materials and methods $(\mathrm{n}=5$ for each group). Lanes: 1. LM9-shCtr, 2. LM9-shANGPTL2, 3. K7M2-shCtr 4. K7M2-shANGPTL2, 5-0S17shCtr, 6-OS17-shANGPTL2. Similar results were obtained by utilizing second shRNA targeting ANGPTL2. Unpaired Student's t-tests, ${ }^{*} p<0.05,{ }^{* *} p<0.01,{ }^{* * *} p<0.001$.

\section{Figure 2}

A

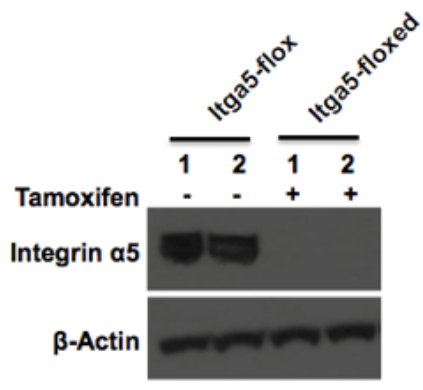

D

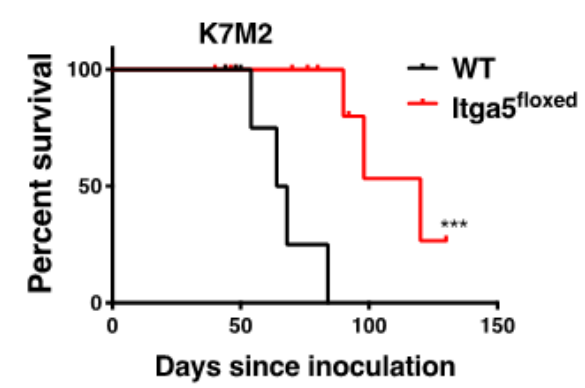

B

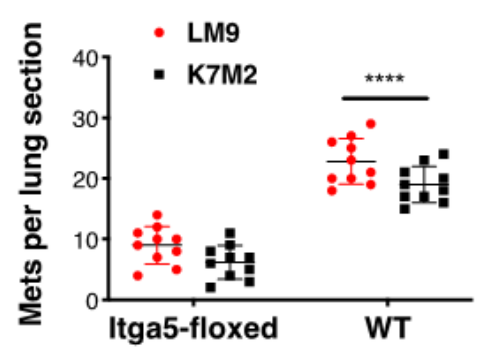

$\mathbf{E}$

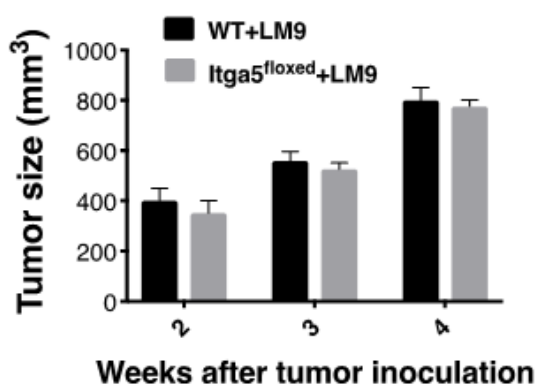

C

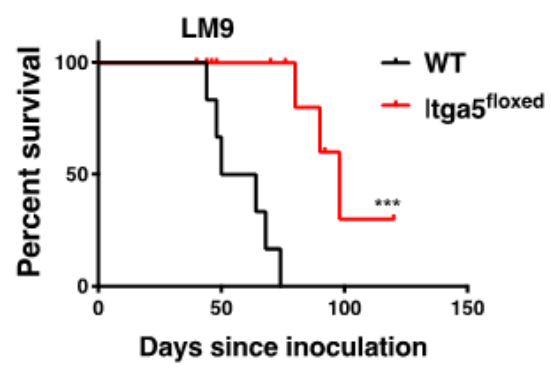

$\mathbf{F}$

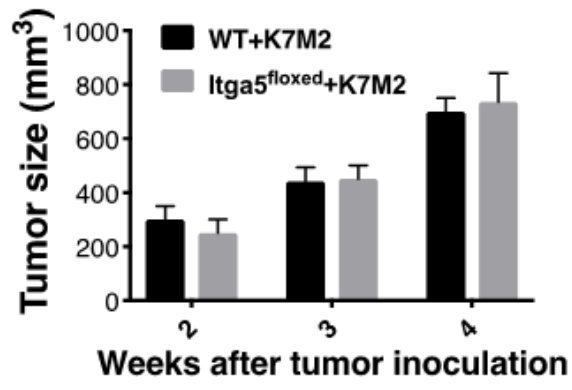

Figure 2 
Integrin a5 $\beta 1$ deficiency in alveolar type II (AT-II) diminishes establishment of osteosarcoma lung metastasis. A. To induce a tissue specific knockout of integrin $5 \mathrm{a}$ in Type II alveolar cells, tamoxifen was administrated. Lung cell suspensions are prepared by intratracheal instillation of dispase and agarose followed by mechanical disaggregation of the lungs. Alveolar type II epithelial cells were purified from these lung cell suspensions through magnetic-based negative selection using a Biotin-antibody, Streptavidin-MicroBeads system. Protein lysate from the purified alveolar type II epithelial cells analyzed by western blot with antibodies as shown. B. Osteosarcoma cells were injected into the tibia of either Itga5-flox (herein, wild-type, WT) or Itga5-floxed mice ( $n=40,10$ mice for each group). After primary tumors reached around $800 \mathrm{~mm} 3$ (range from 4 to 5 weeks), primary tumor containing leg was amputated. Animals reaching endpoints were terminated, and lungs were harvested, insufflated, fixed, sectioned, and stained. The number of lung sections with metastatic nodules were compared with

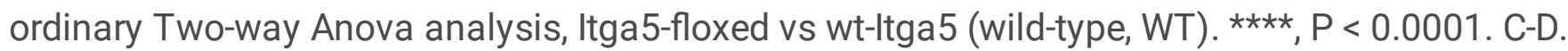
Prolonged survival of mice following the deletion of integrin a5 31 . *ᄎ*, $P<0.001$, WT vs Itga5-floxed injected with LM9 and WT vs Itga5-floxed injected with K7M2 by Log-rank (Mantel-Cox) test. E-F. Deletion of integrin a5 $\beta 1$ in the alveolar type II (AT-II) cells does not impact on the primary tumor growth in the tibia. Tumor growth was monitored by caliper measurements for four weeks.

\section{Figure 3}

A

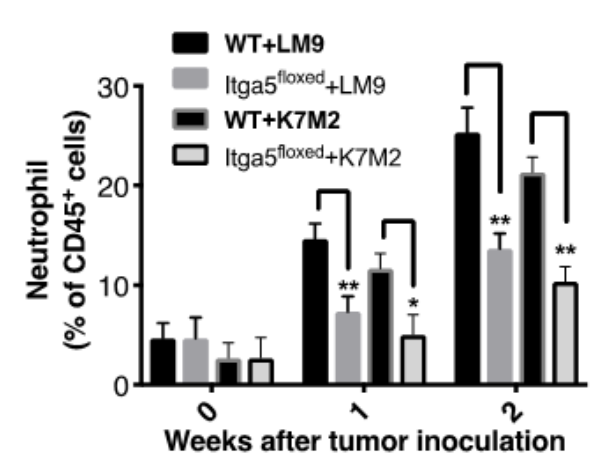

D

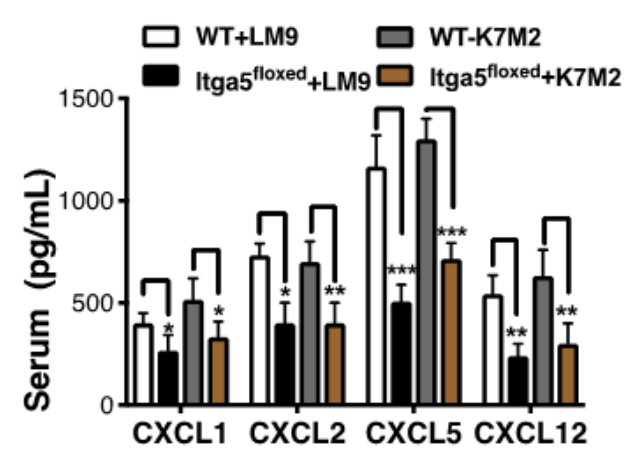

B

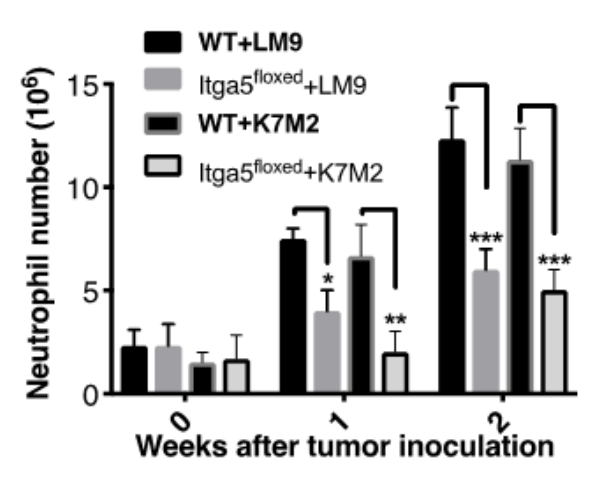

C

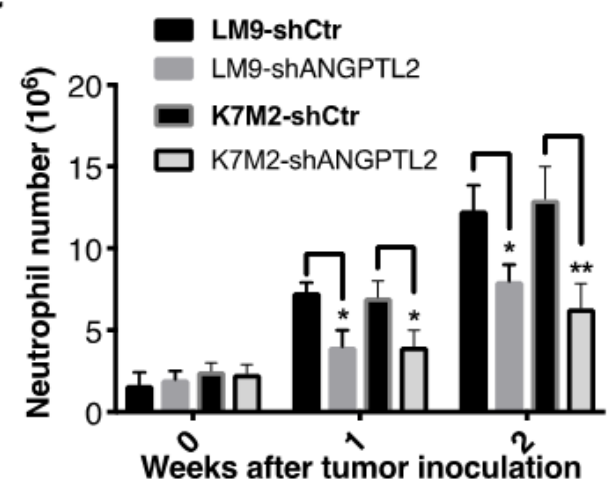

E

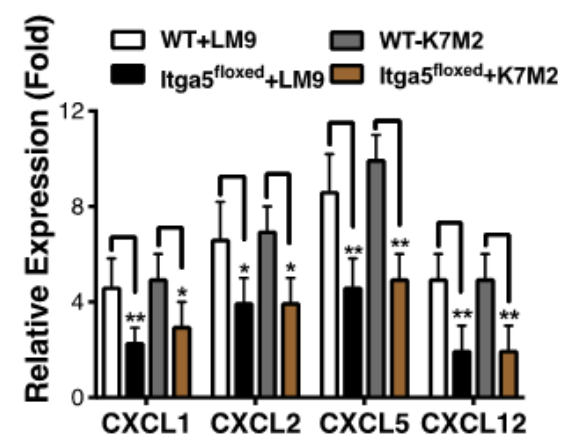

F

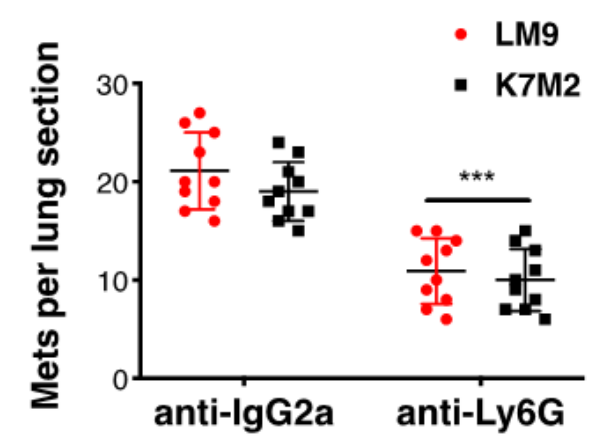

Figure 3 
Integrin a $5 \beta 1$ deficiency reduces neutrophil recruitment to the lung. A-B, The proportions $(A)$ and absolute numbers (B) of neutrophils in the lung was detected by flow cytometry in Itga5-floxed mice or WT littermates after with LM9 and K7M2 cell inoculation into tibia (Gating strategy for neutrophils is shown in Supplementary Figure 3). C. Absolute numbers of neutrophils in the lungs were detected by flow cytometry in WT littermates after intratibial inoculation of LM9-shCtr, LM9-shANGPTL2, K7M2-shCtr and K7M2-shANGPTL2 cells at indicated weeks. D. Serum was isolated from tumor bearing animals one week after intratibial injection of tumor cells and analyzed by ELISA according to the manufacturer's instructions (R\&D Systems). Similar results were obtained in BALF (Supplementary Figure 5A-B) E. CXCL1, CXCL2, CXCL5, and CXCL12 transcripts were significantly lower in Itga5-floxed AT-II cells compared to WT AT-II cells. AT-II cells were isolated from tumor bearing animals after a week of intratibial injection with indicated cells. Subsequently, total RNA was extracted and used for the gene expression analysis of chemokines. Results were normalized to GAPDH. Unpaired Student's t-tests, ${ }^{*} p<0.05,{ }^{*} p$ $<0.01, * \star \star p<0.001$. F. Depletion of neutrophils results in a dramatic reduction of lung metastases. The numbers of lung section with metastatic nodules were compared with ordinary Two-way Anova analysis IG2a vs anti-Ly6G treated. ***, $\mathrm{P}<0.001$.

\section{Figure 4}

A

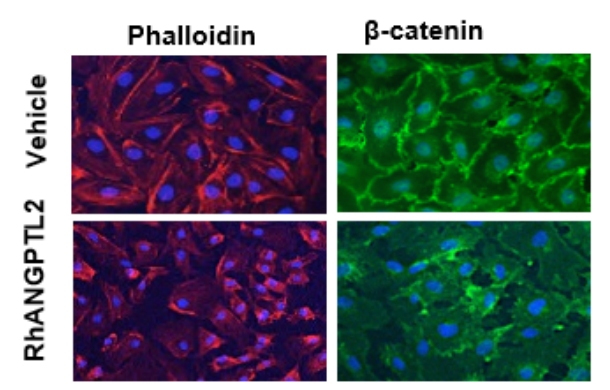

D

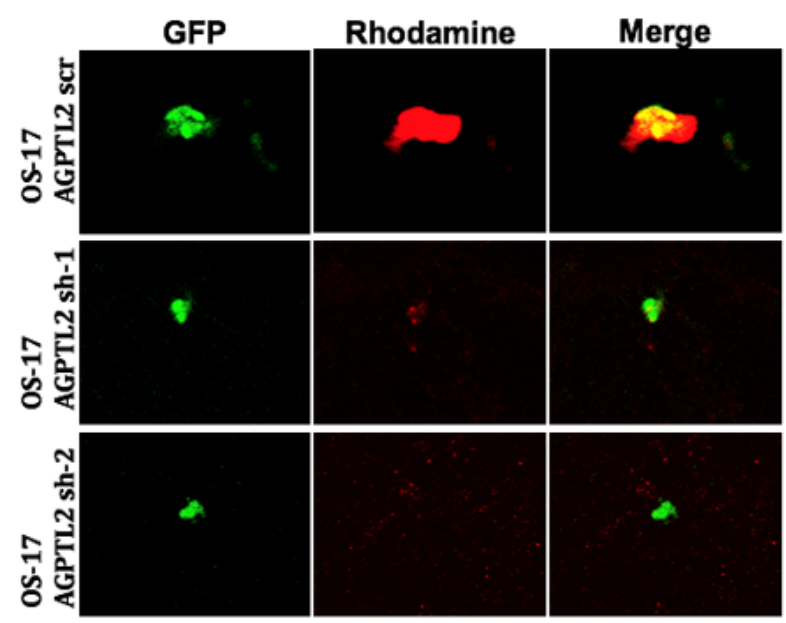

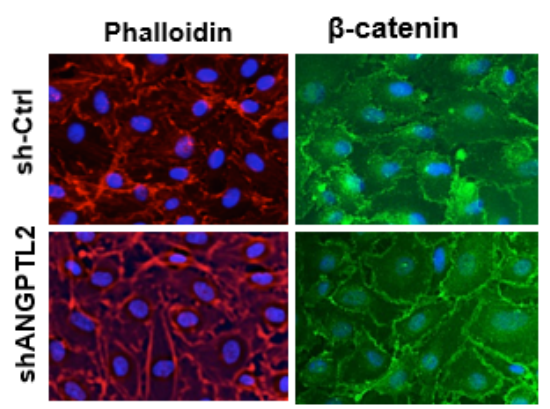

$\mathbf{E}$

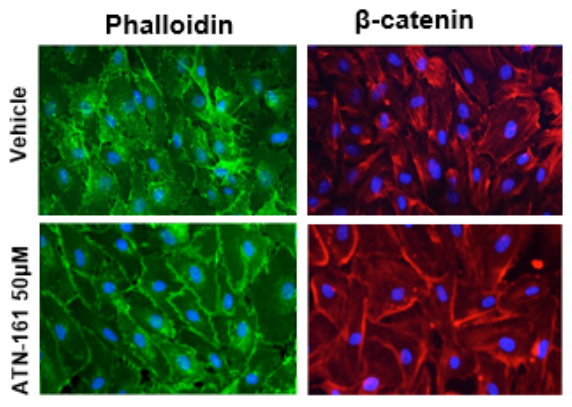

$\mathbf{F}$
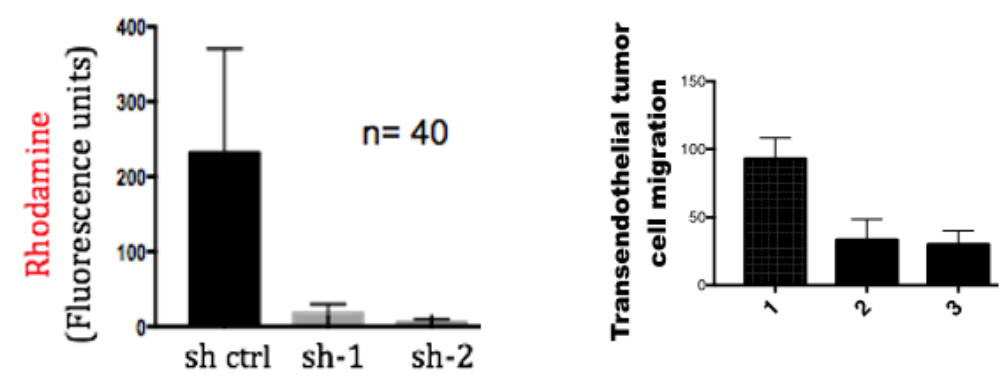

Figure 4 
Tumor-secreted ANGPTL2 promotes osteosarcoma metastasis by mediating endothelial monolayer disruption, lung capillary permeability, and trans-endothelial tumor cell migration. A. HUVEC monolayers were grown to confluence on fibronectin-coated slides and then treated with either vehicle (PBS) or human recombinant ANGPTL2 protein (rhANGPTL2) for 24h. Slides were subsequently fluorescently stained with anti-phalloidin, and anti- $\beta$-catenin antibodies. B. HUVEC monolayers were treated for $24 \mathrm{~h}$ with media conditioned by control OS17 osteosarcoma cells that express ANGPTL2 (ctrl) or knockdown OS17-shANGPTL2 (sh) cells. Samples were stained for $\beta$-catenin and phalloidin. C. Administration of ANGPTL2 receptor inhibitor, ATN-161, prevents disruption of vascular endothelial cell-cell junctions. HUVEC monolayers were treated for $24 \mathrm{~h}$ with media conditioned by OS-17 cells with drug. Subsequently, samples were stained for $\beta$-catenin and phalloidin. D. GFP-labeled indicated OS17 cells were injected via the tail vein and allowed to lodge in the lungs. One-day post injection, a rhodamine-dextran dye was injected into circulation. Three hours after dye injection, lungs were extracted and frozen sections were obtained. Representative confocal images are shown here of cells with and without accumulation of dye in the lung parenchyma. E. Images were obtained as described in (D) with control or ANGPTL2 knockdown OS17 cells. A region of interest was drawn around the GFP-labeled cells and the amount of dextran dye was quantified based on rhodamine emissions. $n=40$ cells; error bars indicate s.e.m; $p$-values calculated using the one-tailed unpaired t-test. F. OS17-shCtr (1), OS17-sh1ANGPTL2 (2) or OS17sh2ANGPTL2 (3) were seeded into trans-well inserts that were previously covered with a HUVEC monolayer. Cells that migrated cross the endothelial layer into the bottom side of the transwell membrane were quantified with Volocity software. $n=15$, error bars indicate s.e.m; $p$-values calculated using the onetailed unpaired t-test. 


\section{Figure 5}

A

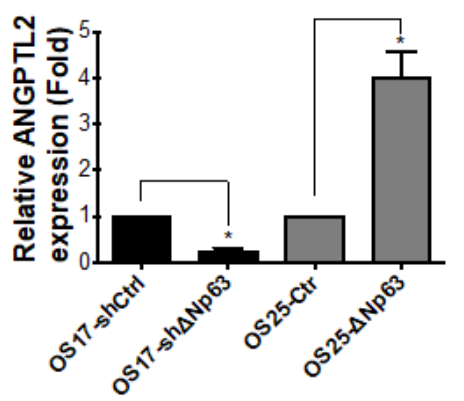

D

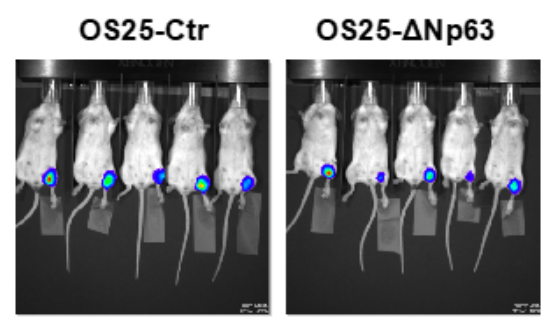

G

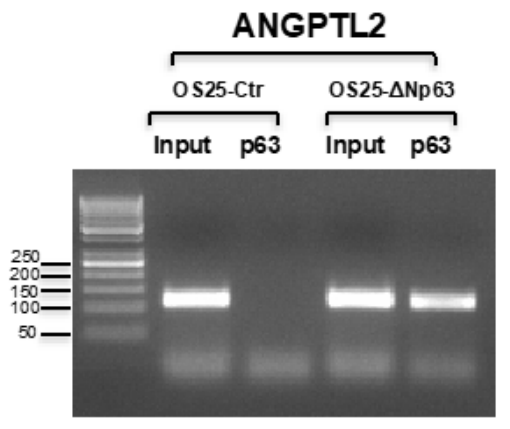

B

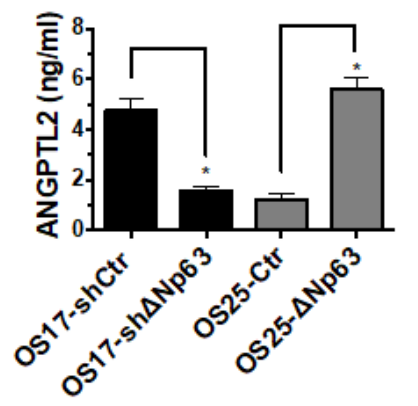

E

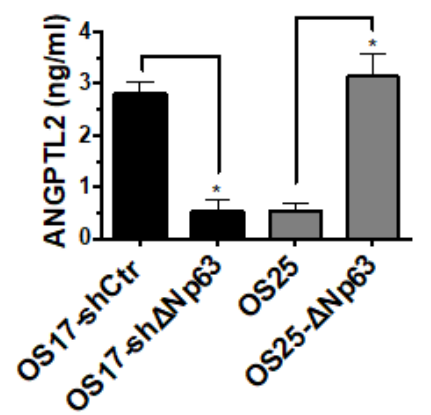

H

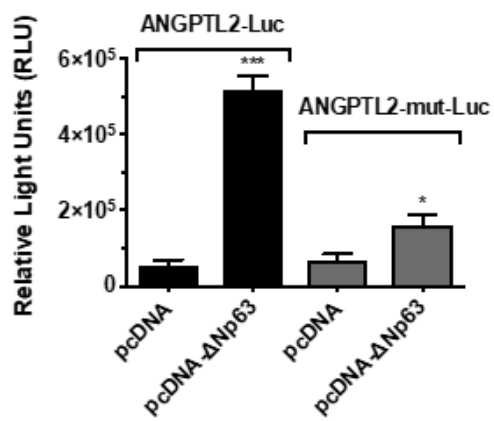

C

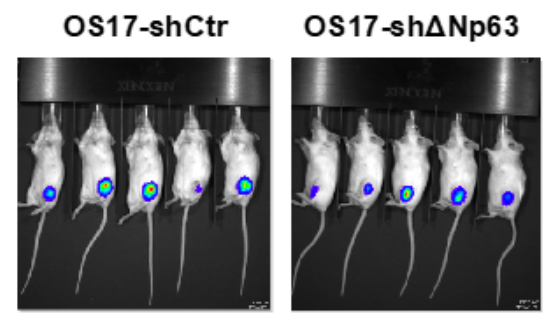

F

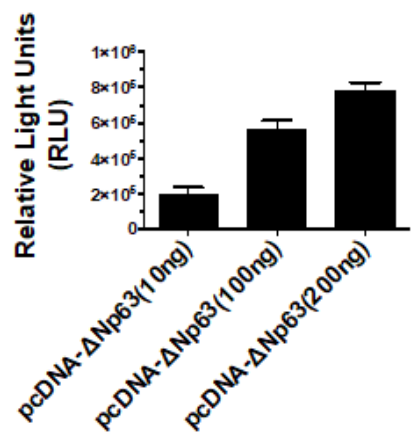

Figure 5

$\triangle$ Np63 induces ANGPTL2 expression and secretion. A. qRT-PCR was used to assess ANGPTL2 mRNA levels in indicated osteosarcoma cells. B. Conditioned medium from indicated cells was collected and used for human ANGPTL2 ELISA (Takara). In all experiments, *P values $<0.05$ was considered significant. C-D. Indicated luciferase expressing osteosarcoma cells was injected into the tibia of SCID mice. A representative bioluminescence image showing inoculated tumor cells after 7 days. E. Serum from tumorbearing animals was obtained post 7 days of tumor inoculation. Subsequently, serum levels of ANGPTL2 were determined using a human ANGPTL2 ELISA kit (Takara). F. ANGPTL2 promoter luciferase construct co-transfected with increasing amounts of $\triangle$ Np63 plasmid into HEK-293 cells. Luciferase assay performed in triplicate wells (mean \pm S.D) $48 \mathrm{~h}$ after transfection as described in materials and methods. G. ChIP assay demonstrates binding of $\triangle$ Np63 on ANGPTL2 promoter containing NF-kB site. Immunoprecipitated samples from indicated cell lines were analyzed by PCR using the primers as described in methods. $\mathrm{H}$. Induction of ANGPTL2 luciferase reporter activity by $\triangle N p 63$ expression in 293T cells correlates well with the presence of NF-KB response elements. ANGPTL2mutNF-KB luciferase 
reporter construct was generated as described in materials and methods and assayed similarly. Unpaired Student's t-tests, * $p<0.05, * \star * p<0.001$ vs control.

\section{Figure 6}

A

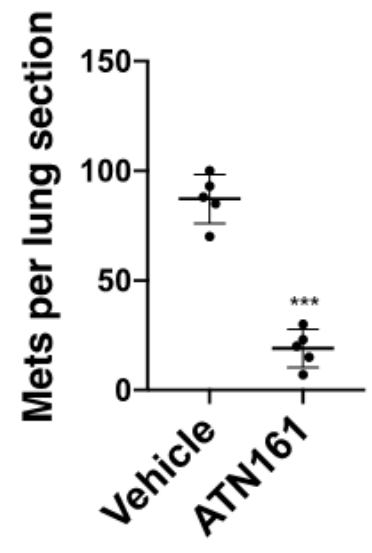

C

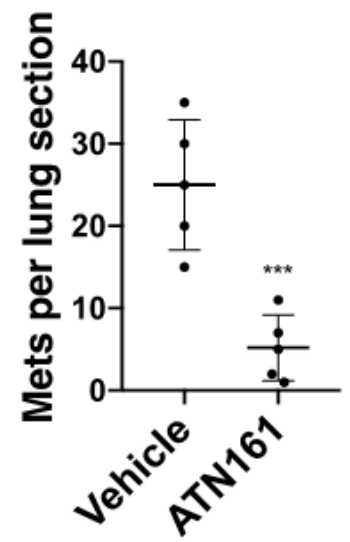

B
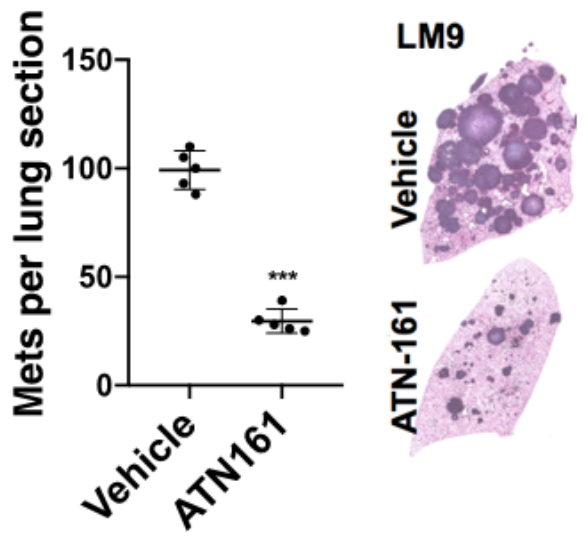

D

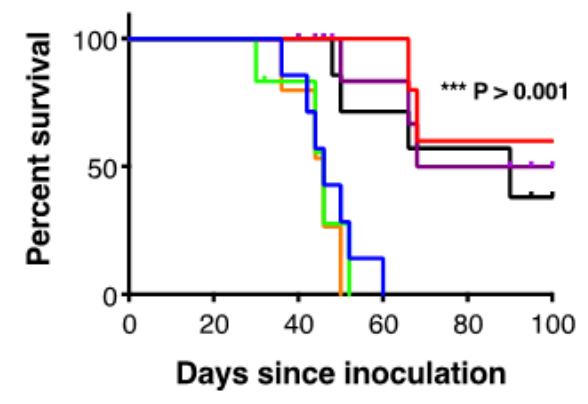

- Vehicle-OS17 - Vehicle-LM9 - Vehicle-K7M2

- ATN161-OS17 - ATN161-LM9 - ATN161-K7M2

Figure 6

ATN-161 treatments significantly lower metastatic burden and increased overall survival. A-C, SCID mice were inoculated with OS-17 cells via tail vein (A), whereas K7M2 (B) and LM9 (C) cells were inoculated in immunocompetent BALB/c mice. Animals were treated with vehicle alone (PBS) or ATN-161 $1 \mathrm{mg} / \mathrm{kg}$ thrice a week) for 6 weeks ( $n=5$, per group). A representative lung section from each group is shown. The

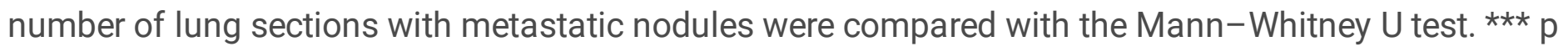
$<0.001$. C. Kaplan-Meier survival analysis of the mice shown. Treatment of ATN-161 was continued post injection until day 42, and stopped. Mice that received ATN-161 (1mg/kg three times a week) experienced significantly better outcomes than vehicle treated group ( $n=10$ mice per group). Mice surviving beyond 100 days were euthanized.

\section{Supplementary Files}


This is a list of supplementary files associated with this preprint. Click to download.

- supplement1.pdf

- supplement2.pdf

- supplement3.pdf 